\title{
Tactile and Kinesthetic Feedbacks Improve Distance Perception in Virtual Reality
}

\author{
Les retours tactile et kinesthésique améliorent la perception de distance en réalité virtuelle \\ Lawrence Makin \\ University of Bristol \\ Bristol, UK \\ lm16720@bristol.ac.uk \\ Gareth Barnaby \\ University of Bristol \\ Bristol, UK \\ gareth.barnaby@gmail.com \\ Anne Roudaut \\ University of Bristol \\ Bristol, UK \\ roudauta@gmail.com
}

\begin{abstract}
Research spanning psychology, neuroscience and HCI found that depth perception distortion is a common problem in virtual reality. This distortion results in depth compression, where users perceive objects closer than their intended distance. Studies suggested that cues, such as audio and haptic, help to solve this issue. We focus on haptic feedback and investigate how force feedback compares to tactile feedback within peripersonal space in reducing depth perception distortion. Our study $(\mathrm{N}=12)$ compares the use of haptic force feedback, vibration haptic feedback, a combination of both or no feedback. Our results show that both vibration and force feedback improve depth perception distortion over no feedback (8.3 times better distance estimation than with no haptic feedback vs. 1.4 to 1.5 times better with either vibration or force feedback on their own). Participants also subjectively preferred using force feedback, or a combination of force and vibration feedback, over no feedback.
\end{abstract}

\section{CCS CONCEPTS}

- Hardware Haptic devices

\section{KEYWORDS}

Haptic; Force Feedback; Tactile Feedback; Depth Perception; Virtual Reality

\section{RÉSUMÉ}

Des recherches en psychologie, neurosciences et IHM ont montré que la distorsion de la perception des distances est

IHM'19, December 10-13, 2019, Grenoble, France

(c) 2019 Copyright held by the owner/author(s). Publication rights licensed to ACM.

This is the author's version of the work. It is posted here for your personal use. Not for redistribution. The definitive Version of Record was published in Actes de la 31e conférence Francophone sur l'Interaction Homme-Machine (IHM'19), December 10-13, 2019, Grenoble, France.

https://doi.org/10.1145/3366550.3372248. un problème courant en réalité virtuelle. Cette distorsion entraîne une compression des profondeurs, et les utilisateurs perçoivent des objets plus proches qu'ils ne le sont. Dans ce papier, nous nous concentrons sur le retour haptique et examinons comment le retour de force se compare au retour tactile pour réduire la compression des profondeurs. Notre étude $(\mathrm{N}=12)$ compare l'utilisation du retour de force, le retour tactile vibratoire, la combinaison des deux ou l'absence de retour. Nos résultats montrent que le retour tactile et le retour de force améliorent la perception de la profondeur. L'estimation de distance est 8.3 fois meilleure que sans retour, par rapport à 1.4-1.5 fois avec retour tactile vibratoire ou de force non-combinés. Les participants ont également préféré utiliser le retour de force, ou une combinaison de force et tactile.

\section{MOTS CLÉS}

Haptic; Retour de Force; Retour Tactile; Perception des distances; Realité virtuelle.

\section{INTRODUCTION}

Inaccurate depth perception in virtual reality, where users incorrectly perceive the depth of virtual objects, is a welldocumented problem and ongoing concern [8]. This distortion is intrinsically linked to the fact that human uses binocular disparities to extract depth information from twodimensional retinal images in stereopsis. And it tends to result in depth compression, i.e. users perceive objects closer than their intended distance and thus affecting virtual reality hardware and content creators alike, particularly for interactive applications such as gaming or training.

Research spanning over 20 years highlights the need for a multimodal approach in solving the problem of depth perception distortion. The vast majority of research appears to revolve around visual cues, such as increasing visual realism [21], removing visual clutter [14], simplifying virtual objects [22] or using the presence of avatars [20]. Yet few studies tackle it through haptic feedback.

Only one series of studies $(\mathrm{N}=4)$ utilized force feedback (using a string-based device) [6]. This study revealed that it was significant in improving depth perception distortion. 
However, as mentioned by the authors, the investigation lacks high level integration between haptics and stereopsis. It is probably due to the fact that the study was conducted 20 years ago with older 3D display technology. The stereoscopic image was displayed on a 120-inch large screen and participants viewed it by wearing liquid-crystalshuttered glasses. They also used simplistic visuals (random dot stereograms). Research of this type have not been replicated with more participants, and with more actual higher-resolution stereoscopic technologies such as virtual reality headsets. This motivates our paper.

To test the hypothesis that haptic feedback improves depth perception in virtual reality, we conducted a study $(\mathrm{N}=12)$ in which we compared force feedback, vibration feedback, and a combination of the two, against no haptic feedback. In particular we looked at depth distortion in the close peripersonal range (within $40 \mathrm{~cm}$ ) which corresponds to the workspace offered by most force feedback desktop devices.

\section{RELATED WORK}

We first discuss work explaining the depth perception distortion. We then go through several general solutions that have been proposed, in particular the ones relating to multisensory integration.

\section{The problem of depth perception distortion}

Prior research shows that a problem [13] exists where humans inaccurately perceive the depth of objects in virtual environments [8]. The reasons are summarized by Lawson et al. [15], which in turn are drawn from Renner et al.'s research [20]. In essence, the problem of depth perception distortion is highly complex, and has yet to be fully resolved. However, strides have been made through improved technology to rectify depth perception distortion. It is generally understood that distance is compressed in virtual environments [8], [13], [25], [21], meaning users tend to perceive virtual objects in relation to themselves (egocentric space) as closer than their actual distance. Similarly, Rolland et al. [22] found that using simple virtual objects such as a cube or a cylinder led to expanded perceptual distortion rather than compressed. It was also found that, in peripersonal space, object distances are more often over-estimated [2].

\section{Solutions to depth perception distortion}

A visual solution to depth perception distortion is to use highly-detailed virtual environments with high resolution rendering to near-replicate the real-world environment. In a study by Ries et al. [21] three separate virtual environments were implemented depicting the same scene, but of different sizes. The authors found that users make accurate judgments of egocentric distance when cognitively 'immersed' or in their terms "accept the virtual environment as being equivalent to the real world”. Kruijff et al. [14] discovered that visual clutter can worsen the effects of depth distortion, which is in stark contrast to Ries et al.
Another method to reduce depth perception distortion involves physically walking. Kelly et al. found perception of object size and distance was improved due to the constant visual feedback in relation to changing location in space [25]. In large virtual environments, and provided users have real world space in which to 'walk' virtually, this approach could be useful. However, this cannot be assumed, and could pose a danger in terms of real world objects obscuring the scene travelled in the virtual environment.

The advent of modern stereoscopic virtual reality headmounted displays (HMDs, such as the Oculus Rift and HTC Vive) has allowed for improved depth perception in virtual environments [13]. The improvement of depth perception distortion is potentially to the point that the HMDs provide "immersion and depth perception on a level that proved sufficiently realistic for healthy young adults to perform natural reaching movements" [9]. Yet Lin et al. suggest that stereoscopic depth cues could be part of the problem of depth perception distortion [16]. These conflicting views could mean that depth perception distortion is harder to solve through HMDs, and thus visual stimuli, alone. Grossman et al [10] also demonstrate that depth perception is improved when using a volumetric display compared to 3D displays. This result also potentially translate to levitated displays, e.g. [19] as users can see real objects.

\section{Solutions using multisensory feedback}

Further research has found that the use of combined, or multimodal, sensory information (visual, aural, tactile) improves virtual environment presence and immersion [23], [12] and has a positive effect on depth perception [15], [9]. Hecht and Reiner suggest that audio and haptic stimuli are dominated by visual stimuli when only one or the other is coupled with the visual stimuli, but not both [11] and are more likely to go unnoticed. They also note that when all three modalities are present together, any individual modality can dominate depending on its intensity compared to the other modalities. According to Lawson et al. there is also the possibility that multiple stimuli could conflict and thus reduce spatial awareness [15]. This claim is further backed up by a study in the field of neuroscience, in which it was found that visual stimuli could be suppressed by accurate haptic stimuli [17].

These could be seen as a conflicting standpoint compared to that of Cooper et al. [7], who claims that auditory and tactile cues enhance the sense of immersion in virtual reality. However, it is worth pointing out that Hecht and Reiner's methodology implemented a non-virtual environment setup with at least decade-old technology, which could be behind the discrepancy. Conversely, Rosa et al. conclude that visual cues can have an effect on how users perceive vibrotactile feedback, albeit not necessarily accurately [23]. It is possible that audio cues can solve the problem of depth perception distortion through off-setting their spatial location in relation to the appropriate visual cue(s) [8]. In other words, the audio cues are mapped 
differently to what would be expected in the real world. But with such conflicting views on the dominance of the various stimuli versus auditory and tactile stimuli improving virtual environment experience in tandem, it is difficult to put haptic stimuli in perspective.

Cooper et al. also state that auditory and tactile cues are useful when participants need to reach and grasp objects during the virtual reality interaction [12]. On the basis of this, they designed a virtual environment in which participants reach for and touch a virtual cube using a Phantom haptic device combined with a vibration motor.

Haptics, or tactile interaction, as a modality in virtual environments is under-researched in comparison to others [15], [7], [3]. Azmandian et al. note that "studies have shown that using haptics can lead to significantly increased presence and spatial knowledge training transfer" [3]. Research since at least Bougila et al. [6] provides evidence for haptic feedback as a solution to depth distortion, but since then, very little research has been done in this area. Various forms of haptic feedback, such as passive, vibration, and force devices, have also been created since then.

\section{EXPERIMENTAL DESIGN}

The goal of this experiment is to investigate if haptic feedback enhances depth perception distortion, i.e. reduces the error made in estimating distance. Our secondary goal was to quantify the effects of different haptic conditions on depth perception distortion. We compared four conditions: tactile, force feedback, a combination of the two, and a nonhaptic baseline. We now describe the choice behind the experimental design and give details about our study implementation before discussing the results.

\section{Apparatus}

Figure 1 shows the haptic apparatus. We used a Phantom Premium haptic device to provide force feedback at a fingertip. We added a 3D printed finger rig to allow the participants to use their finger rather than the classical pen end-effector provided (Figure 2) in order to better represent direct manipulation tasks. The Phantom can also provide vibration feedback via its internal motors, although it does not focus on the fingertip of the user but instead transfers the vibration down the shaft of the haptic arm. Thus, we used a small disc vibration motor mounted at the end of the phantom end effector (Figure 3) to produce better vibrations. The vibrations were controlled by an Arduino Mega 2560 along with a Grove System vibration motor. We used an HTC Vive and a Leap Motion for tracking user's hands. We used Unity and a 3rd-party plugin from the Unity Asset Store allowing the Phantom to communicate its movements to Unity through its OpenHaptics Toolkit (Unity 5 Haptic Plugin for Geomagic OpenHaptics).

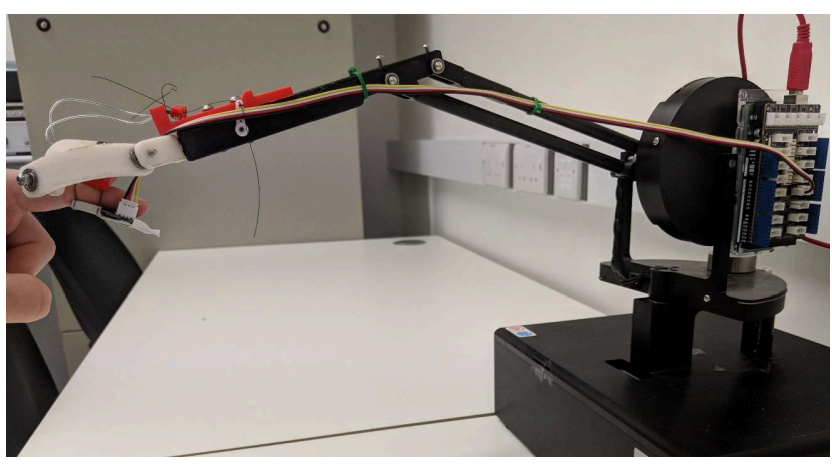

Figure 1. Overview of the apparatus.

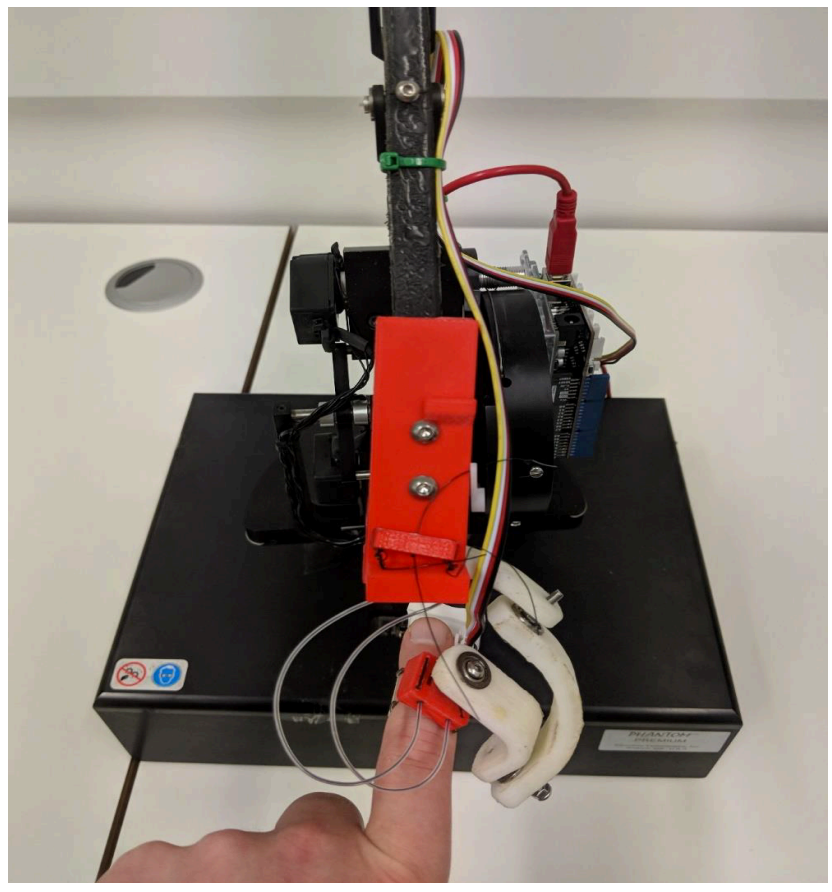

Figure 2. The 3D-printed finger rig with gimbal and finger clamp, used with the Phantom Premium haptic device.

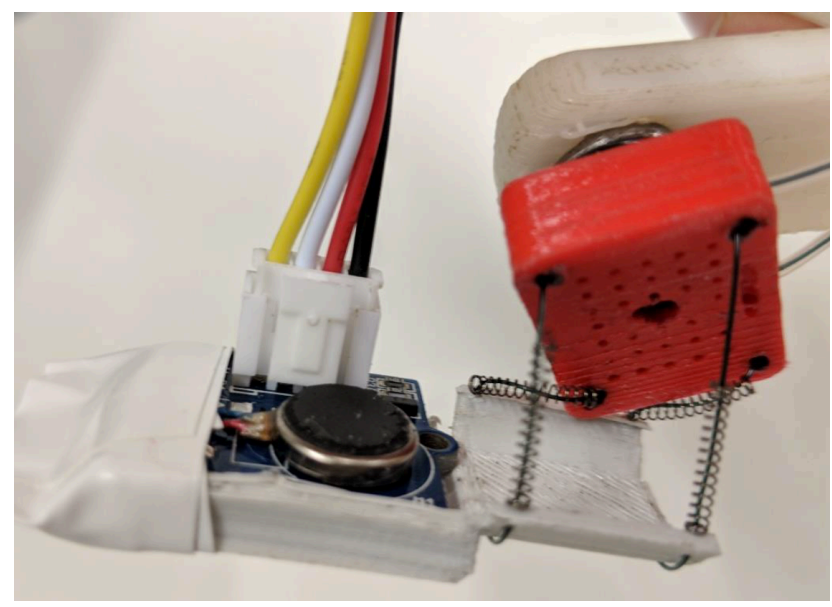

Figure 3. The vibration motor used at the fingertip. 


\section{Virtual environment}

Participants touched a virtual cube (Figure 4). We chose this shape following Rolland et al.'s assertion that simple shapes, such as cubes and cylinders, in virtual environments tend to be perceived as further away [22] than the object's actual distance. We used a hand model from the Unity Asset Store to portray a sense of presence to the participants, rather than use the default virtual stylus provided with the OpenHaptics Toolkit Unity port (Figure 4). In Renner et al.'s review of egocentric distance perception in virtual reality [20], there is evidence to suggest that such a presence of an avatar aids in reducing depth distortion.

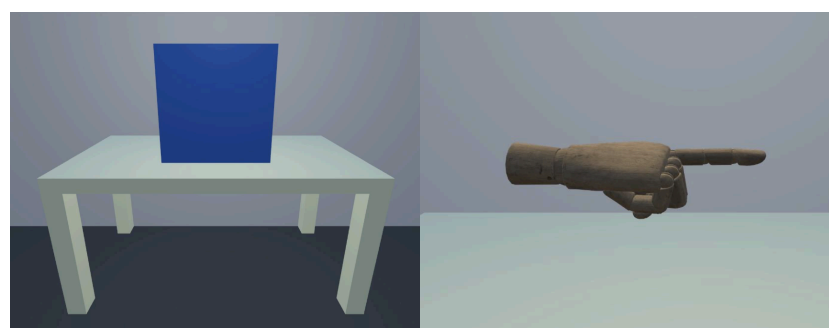

Figure 4. The virtual environment used in the user study: (left) a $50 \mathrm{~cm}$ cube on top of a $140 \mathrm{~cm}(W) X 72 \mathrm{~cm}(\mathrm{H}) \mathrm{X}$

$80 \mathrm{~cm}(\mathrm{D})$ desk created in Blender; (right) a virtual hand model posed in the general shape expected from participants' hands once in the Phantom's finger rig.

\section{Task}

Our task was based on Bouguila et al. [6] reaching experiment. Bouguila et al. designed a reaching experiment utilizing the big SPIDAR [24] haptic device, where a seated participant would align their hand with where they perceived a virtual square rendered in front of them in a $3 \mathrm{D}$ display. Participants would state when they thought they had reached the square, and the distance travelled by their hand would be recorded at this point. The square was moved between tasks, and participants were asked to close their eyes briefly between tasks to prevent training.

We developed a series of eight reaching zones (Figure 5). Each dot represents a reaching task. The black dots represent the central point location of the bottom edge of the visible virtual cube's front face. The red dots represent the same, except for the cube being invisible in this case, with the corresponding visible cube still in place behind the red dot. The dots to either side of the central ones are offset by 45 degrees in the forward direction, to prevent training.

The distance between the red and black dots in the forward position is $10 \mathrm{~cm}$. This is the distance we arbitrarily chose to test for depth perception against the visible cube at any given position. We choose $10 \mathrm{~cm}$ because we wanted to have a large enough distance but not too large to fit within the dimension of our apparatus. For each reaching task, we apply one of four feedback types: no haptic feedback (as a control), vibration feedback, force feedback, and a combination of vibration and force feedback. If depth perception distortion exists at $10 \mathrm{~cm}$, participants should at least occasionally feel as though they are touching the visible cube, even though they are $10 \mathrm{~cm}$ in front of it when a haptic force is applied.

\section{Procedure}

The participants were sitting at a table. They were asked to move the hand they had placed in the rig as close to themselves as the Phantom would allow. We manually guided their arm to ensure the Phantom arm was as straight as possible for the task. They practiced with the system. When a trial started, the participants first had to close their eyes. The virtual cube would then appear in a predefined pseudo-random position, at which time we asked the participant to open their eyes and reach towards the center of the cube's front face. We specifically asked this as if a participant reached around the edges of the cube, they would gain more perspective of whether or not they had touched its surface. If a force was applied, either on contact with the cube, or $10 \mathrm{~cm}$ before it (unbeknownst to the participant), we asked the participants if they thought they had touched the cube. We then asked them to hold their hand steady so that we could submit their distance data and answer via another button in the Unity scene. We then asked the participant to close their eyes, at which point we repeated the process, until all 32 tasks had been completed. The haptic device arm was moved back to its original position after each trial.

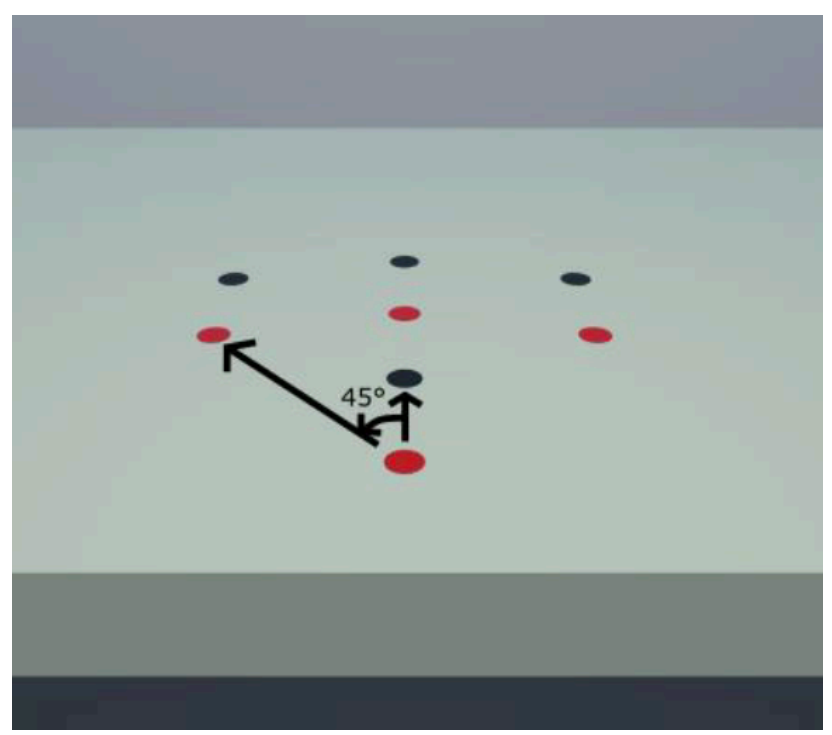

Figure 5. The black dots represent the mid-point of the front face of the cube for each reaching task. The red dots are 'fake', invisible cube where forces are applied $10 \mathrm{~cm}$ in front of the visible cube.

\section{Counterbalancing}

We randomized the order of positions for each haptic feedback, so that the order between the sets was not the same, to prevent training. We used a Latin square to counterbalance the presentation of the feedback (no haptic 
feedback, force feedback, vibration and force feedback, vibration feedback). As there are 4 feedback types and 8 dot positions, that means a total of 8 sets of 4 tasks, or 32 tasks per participant. The study lasted for $\sim 30$ minutes including breaks that could be taken between conditions.

\section{Dependent variables}

Each participant was asked to stop moving the Phantom arm at the point they felt they were touching the virtual cube (if no haptic force was applied) or they felt a haptic force being applied. They were then asked if they thought they had reached the virtual cube or if they were experiencing the haptic force too early. The experimenter clicked on a button within the Unity scene to submit the participant's answer, and at that moment the distance travelled by the Phantom arm, the distance of the virtual cube from the participant, and the current haptic feedback type, were logged. The raw data per participant included the distance they reached per task, the distance of the actual front face of the virtual cube, and whether or not the participant thought they'd success-fully reached and touched the front face of the cube.

We also designed a post-study questionnaire with answers on 7-point Likert scales. Each question asked the participant their opinion of a specific aspect of the interactive part of the study with answers ranging from "Strongly Disagree" to "Strongly Agree". The purpose was to determine whether there is any subjective user preference of the four methods of haptic feedback provided during the reaching tasks.

\section{Calibration}

To ensure the virtual environment was calibrated to be as accurate as possible in terms of scale and rotation, we performed calibration of the Phantom arm and the Vive headset for each participant. Calibration of the Phantom is relatively straightforward as it utilizes its own software. For the calibration of the Leap Motion we asked each participant to grasp the edge of the real desk in front of them and see if the Leap Motion hand tracking models lined up with the virtual desk. It could take a few attempts to make sure the participants were seated close enough and central to the midpoint of the real desk to reduce inaccuracy of their position in relation to the virtual desk.

Note that the HMD, Leap Motion, and Phantom have their own coordinate frames. We manually aligned these and hardcoded the offsets in software. Note that we found that the leap motion tracking was inconsistent between users and that an offset was detected. We had to realign the Leap Motion coordinate frame with the HMD and the Phantom for each user. We also observed that the phantom can impact the Leap Motion tracking in some capacity. However, it only affects the finger attached to the Phantom and not the others finger and the hand which can be used to ensure a good tracking. We also did some informal test and found that the Phantom interferes with tracking when curling the finger. In this case the Leap Motion detects it as being outstretched or partially curled. But this did not cause issues in our study as the finger was kept outstretched during the entire experiment.

\section{Participants}

12 participants (2 female) (1 left-handed, 1 ambidextrous) (from 20 to 35 years old) were recruited in our institution and received no intensive for their participation. They had never used an actuated arm nor a force feedback device before but were familiar with virtual reality technologies.

\section{RESULTS AND DISCUSSION}

Of the 384 total tasks (32 tasks per participant), we filtered out the mistakes and excluded them from the analysis. The mistakes occurred when participants failed to keep their reaching hand steady at the point of contact. In total, the mistakes rate was 10 , or approximately $2.6 \%$ of all tasks.

\begin{tabular}{|c|c|c|c|c|c|c|c|c|}
\hline & \multirow[t]{2}{*}{ None } & \multicolumn{2}{|l|}{ Vibration } & \multicolumn{2}{|l|}{ Force } & \multicolumn{2}{|c|}{ Vibration + Force } \\
\hline & & & Reached & $\begin{array}{l}\text { Not } \\
\text { Reached }\end{array}$ & Reached & $\begin{array}{l}\text { Not } \\
\text { Reached }\end{array}$ & Reached & $\begin{array}{l}\text { Not } \\
\text { Reached }\end{array}$ \\
\hline \multirow[t]{2}{*}{$\mathrm{N}$} & Valid & 96 & 44 & 49 & 47 & 47 & 40 & 53 \\
\hline & $\begin{array}{l}\text { Mis- } \\
\text { sing }\end{array}$ & 0 & 52 & 47 & 49 & 49 & 56 & 43 \\
\hline \multicolumn{2}{|c|}{ Mean } & -.0281 & -.0197 & -.1669 & -.0192 & -.0927 & -.0034 & -.0884 \\
\hline \multicolumn{2}{|c|}{$\begin{array}{l}\text { Std. err } \\
\text { of mean }\end{array}$} & .00693 & .00348 & .01225 & .01025 & .00497 & .00155 & .00723 \\
\hline \multicolumn{2}{|c|}{ Median } & -.0141 & -.0120 & -.1231 & .0000 & -.1000 & .0000 & -.1000 \\
\hline \multicolumn{2}{|c|}{$\begin{array}{l}\text { Std. } \\
\text { deviation }\end{array}$} & .06788 & .02308 & .08578 & .07028 & .03407 & .00977 & .05267 \\
\hline
\end{tabular}

Figure 6. Descriptive statistics for the quantitative user study data, measuring the difference in distances recorded in meters between the virtual cube and where the participants thought the cube was while trying to touch it.

\section{Quantitative results}

Figure 6 shows the overall data gathered for each type of feedback. We used non-parametric tests since a ShapiroWilk test did not validate the assumption of normality. We ran a Friedman test to determine if there were differences in distances recorded when participants felt they had touched a virtual cube when a haptic force was applied (vibration, force feedback, and a combination of the two) versus no force applied. Pairwise comparisons were performed with a Bonferroni correction for multiple comparisons. There was a significant difference in distances measured between vibration feedback and all other feedback types, $\chi \mathbf{2}(3)=\mathbf{3 4 . 4 9 6}, \boldsymbol{p}<\mathbf{. 0 0 0 5}$. Post hoc analysis revealed statistically significant differences in distances recorded with vibration feedback applied $(\mathbf{M d n}=$ -0.012) and no haptic feedback $(\mathbf{M d n}=\mathbf{- 0 . 0 1 4 1})(\boldsymbol{p}<\mathbf{. 0 0 0 5})$, force feedback $(\mathbf{M d n}=\mathbf{0 . 0})(\boldsymbol{p}<\mathbf{. 0 0 0 5})$, and combined force and vibration feedback $(\mathbf{M d n}=\mathbf{0 . 0})(\boldsymbol{p}<\mathbf{. 0 0 0 5})$ respectively. No significance was found between any comparison of no haptic feedback, force feedback, and combined feedback. 
We ran a second Friedman test on the cases where participants felt they hadn't touched the virtual cube, to see if there is any difference in distances over the different feedback types. Pairwise comparisons were performed with a Bonferroni correction for multiple comparisons. There was a significant difference in distances measured between the fake point of contact of the cube when vibration is applied too soon, and the fake point of contact of both force feed-back and the combined feedback, $\chi 2(2)=41.738, p<$ .0005. Post hoc analysis revealed statistically significant differences in distances recorded with vibration feedback applied too early $(\mathbf{M d n}=\mathbf{- 0 . 1 2 3 1})$ and force feedback (Mdn $=-.1)(p<.0005)$, and combined feedback $($ Mdn $=-.1)(p<$ .0005) respectively. No significant difference was found between force feedback and combined feedback distances when the forces were applied too soon.

\section{Summary of the quantitative results}

Our results confirm that haptic force feedback does significantly improve depth perception distortion when compared to no haptic feedback. We also found that a combination of vibration feedback and force feedback does significantly improve depth perception distortion compared to no haptic feedback. Overall we found that with force feedback or vibration only, participants estimated distances around 1.4 to 1.5 times better than with no haptic. But with both feedbacks combined they were able to estimate distance 8.3 time better than with no haptic.

Note that in both sets of reaching tasks involving force feedback, the median distance travelled tended towards the exact point of contact of the surface of the virtual cube $(\mathbf{M d n}=\mathbf{. 0})$ or the 'fake' cube $(\mathbf{M d n}=\mathbf{. 1})$, depending on where the force was applied. In contrast, the medians for both vibration feedback (Mdn $=$-.0120) and no haptic feedback $(\mathbf{M d n}=-\mathbf{0 1 4 1})$ tended towards $-1.5 \mathrm{~cm}$, in other words beyond the front surface of the virtual cube.

In the data where participants felt they had not reached the virtual cube when a force was applied, we found a significant difference in accuracy of vibration feedback compared to both force feedback and the combination feedback. This is not unexpected, as in both sets of reaching tasks involving force feedback, participants could not move the Phantom arm beyond the 'fake' cube surface. Conversely, nothing was in place to stop participants from moving through the same 'fake' cube with only the vibration feedback active, allowing for a discrepancy in distance travelled.

\section{Subjective results}

Figure 7 shows the results of the post-questionnaire. The three last questions (displayed on the right) were general questions: "Did you feel that force feedback felt more realistic compared to vibration feedback? Did you enjoy your virtual reality and haptic feedback experience? Do you think virtual reality could benefit from haptic feedback?". We asked these questions in order to ensure that none of our participants had issues with the overall set-up and to have an overall opinion of the feeling about force feedback and vibration feedback. Our results confirm that our participants had a good experience and had a positive feeling about the haptic feedback used in the study.

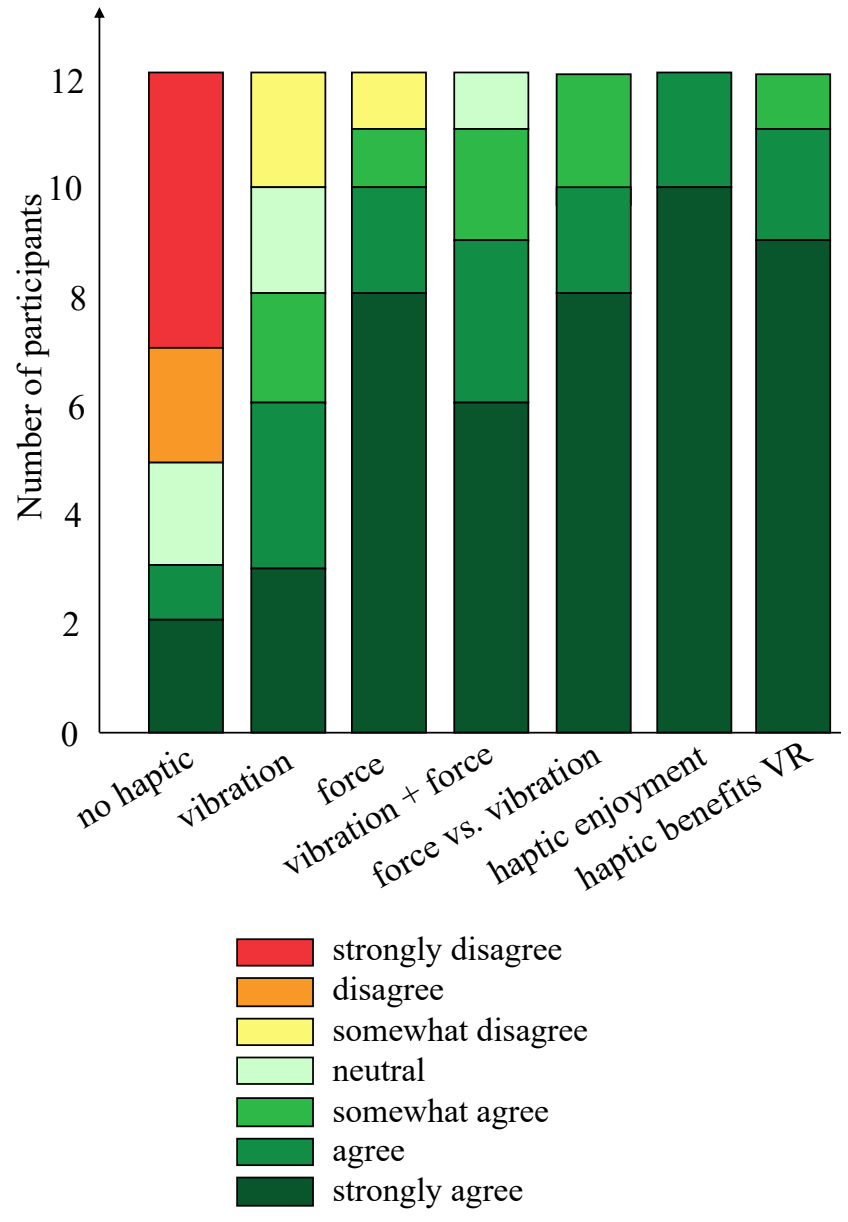

Figure 7. Stacked bar graph representation of the questionnaire data.

We then looked at the four first questions which are comparable: "Did you feel that (no haptic, vibration, force feedback, both) feedback made it easy to touch the cube (4 questions)?". Our questionnaire produces non-parametric ordinal data, so we looked at them using a non-parametric Wilcoxon signed-rank test. However, testing the difference of values between question variables yielded an asymmetrical distribution. We thus began by using a Spearman's Rank Order Correlation, but scatter graph plots showed that the variable relationships were not monotonic. Thus, we used a Sign Test. We compared the first four questions in pairs as seen in the following.

\section{Vibration feedback vs. no haptic feedback}

Of the 12 participants, 9 felt vibration feedback made touching the cube easier, 2 felt no haptic feedback was easier, and 1 felt there was no difference between the two. Overall, participants felt that vibration feedback made 
touching the cube easier $(\mathbf{M d n}=\mathbf{5 . 5})$ compared to no haptic feedback (Mdn $=\mathbf{2 . 0})$, a statistically insignificant increase in the median differences of $3.5, \boldsymbol{p}=\mathbf{. 0 6 5}$.

\section{Force feedback vs. no haptic feedback}

9 participants felt force feedback made touching the cube easier, 1 felt no haptic feedback was easier, and 2 felt no difference between the two. Participants felt that force feedback made touching the cube easier $(\mathbf{M d n}=\mathbf{7 . 0}) 37$ compared to no haptic feedback $(\mathbf{M d n}=\mathbf{2 . 0})$, a statistically significant increase in the median differences of $5, \boldsymbol{p}=\mathbf{. 0 2 1}$.

\section{Force feedback vs. vibration feedback}

6 participants felt force feedback made touching the cube easier, 1 felt vibration feedback was easier, and 5 felt there was no difference between the two. Overall, participants felt that force feedback made touching the cube easier (Mdn $=7.0)$ compared to vibration feedback $(\mathbf{M d n}=\mathbf{5 . 5})$, a statistically insignificant increase in the median differences of $1.5, \boldsymbol{p}=\mathbf{0 . 1 2 5}$.

\section{Combined feedback vs. no haptic feedback}

9 participants felt the combination of force and vibration feedback made touching the cube easier, 2 felt no feedback was easier, and 1 felt there was no difference between the two. Overall, participants felt that the combined feedback made touching the cube easier $(\mathbf{M d n}=\mathbf{6 . 5})$ compared to none $(\mathbf{M d n}=\mathbf{2 . 0})$, a statistically significant increase in the median differences of $4.5, \boldsymbol{p}=\mathbf{. 0 2 1}$.

\section{Combined feedback vs. vibration feedback}

8 participants felt the combination of force and vibration feedback made touching the cube easier, 0 felt vibration feedback was easier, and 4 felt there was no difference between the two. Overall, participants felt that the combined feedback made touching the cube easier $(\mathbf{M d n}=$ 6.5) compared to vibration (Mdn $=5.5)$, a statistically significant increase in the median differences of $1, \boldsymbol{p}=\mathbf{. 0 0 8}$.

\section{Combined feedback vs. force feedback}

4 participants felt the combination of force and vibration feedback made touching the cube easier, 4 that force feedback was easier, and 4 felt no difference between the two. Overall, participants felt that the combined feedback did not make touching the cube easier $(\mathbf{M d n}=\mathbf{6 . 5})$ compared to force (Mdn $=7$ ), a statistically insignificant decrease in the median differences of $0.5, \boldsymbol{p}=\mathbf{1 . 0 0 0}$.

\section{Summary of qualitative results}

Vibration feedback was not seen to be significantly better than no haptic feedback in the ease of touching a virtual cube, while force feedback did show significance $(\boldsymbol{p}=\mathbf{. 0 2 1})$ over no haptic feedback, as did the combined haptic feedback $(\boldsymbol{p}=\mathbf{. 0 2 1})$. However, there was no statistical significance in force vs. vibration, while there was significance in the combined haptic feedback vs. vibration. Yet there was no significance between the combined haptic feedback and force feedback whatsoever $(\boldsymbol{p}=\mathbf{1 . 0 0 0})$.
This outcome seems to show that there is a subtle difference in preference of haptic feedback types, with a general trend towards either force feedback or a combination of vibration of force feedback. We can draw the conclusion that vibration is not preferred over no haptic feedback, and that force feedback is preferred over no feedback. There is no significant difference in preference between force feedback and the combined force and haptic feedback. Participants enjoyed the haptic experience, generally felt it was beneficial to virtual reality, and felt force feedback is more realistic compared to vibration feedback. Thus, in our opinion force feedback is the common factor in haptic preference to touch virtual objects, and from a purely interactive enjoyment aspect should be the method to use going forward.

\section{DISCUSSION}

Haptic feedback does improve depth perception in virtual reality, but we did not find that force feedback does significantly improve it compared to vibration feedback. The fact that there was still depth distortion expansion (albeit only around 1 to $1.5 \mathrm{~cm}$ ) for both vibration feedback and no haptic feedback tasks, suggests that both Naceri et. al [18] and Rolland et al. [22] are correct in their assessment of depth distortion expansion in short distances, and simple objects such as cubes, respectively. Furthermore, it could be argued that using the hand model avatar could have reduced depth perception distortion in the experiment, as suggested by Renner et al. [20].

There was a significant difference in force feedback compared to vibration and the combination of feedback when the force was applied at $10 \mathrm{~cm}$ before the surface of the cube. This may indicate that vibration introduces a certain level of inaccuracy in depth perception, as in comparison the distances travelled under force feedback conditions was very accurate. This is unsurprising considering the arm stops almost completely when touching a virtual object with force feedback. Our qualitative analysis indicated a preference for force feedback or combined force over no feedback in terms of how easy the haptic feedback made touching a virtual cube. There was also a strong opinion in favor of using haptic in virtual reality experiences.

\section{Implications}

Our results have implication for the design of haptic system. Currently most haptic systems are designed with tactile (vibration) or force feedback. New force feedback systems are emerging every year (e.g. the Mantis force feedback system [4]) and our study suggests that there is a benefit in combining force feedback with some form of tactile feedback.

In addition, haptic force feedback devices are particularly used in applications requiring high-precision haptic rendering such as teleoperation, medical training and of 
IHM'19, December 10-13, 2019, Grenoble, France

course gaming in immersive environments, in order to offer users, the ability to touch and sense in the digital world. Being able to enhance the perception of distance and thus increase the accuracy of such task is thus, we believe, a substantial insight for designers and researchers working within those areas.

\section{Limitations}

One limitation of our study is the short range of the Phantom (40 cm with the 3D-printed finger rig). This may be one of the reasons why depth distortion when reaching with no haptic feedback was small (around $2 \mathrm{~cm}$ away from the surface on average). This short range compressed the usable depth into about $20 \mathrm{~cm}$, as there needed to be free space both before and behind the virtual cube and the 'fake' virtual cube at any reaching task position. Without the free space, participants would not be able to move the Phantom arm forward during the closer reaching tasks. We think a haptic device such as the LHIfAM [5] would be better, as its range of forward depth only starts around $30 \mathrm{~cm}$ but continues on to $1.5 \mathrm{~m}$, which we believe would extrapolate the depth distortion effects found in the study.

One of the participants mentioned that the vibration feedback could be more realistic if it was briefer rather than continuous. We choose to produce the vibration in the same manner as the Phantom's force feedback, i.e. continuous as long as a collision is detected. The potential issue with this is that the user will feel a vibration at any point within the collider of the cube, which is the cube's entire volume. This could explain why the travelled distances during the vibration reaching tasks were the least accurate. Participants continued moving the virtual hand forward as no force was stopping them, yet vibration continued. It is possible that this made them believe they were still touching the surface of the cube rather than moving inside it. Further studies thus need to investigate different types of vibration patterns.

\section{CONCLUSION}

Our work investigates the effect of haptic feedback on the improvement of depth perception distortion in virtual reality. Our study using a virtual reality headset and four different types of haptic feedback demonstrates that haptic modality improves depth perception. With force feedback or vibration only, participants estimated distances around 1.4 to 1.5 times better than with no haptic; but with both feedbacks combined they were able to estimate distance 8.3 time better than with no haptic. We also found that participants subjectively preferred using force feedback, or a combination of force and vibration feedback, over no feedback. We think our work can benefit to virtual reality hardware and content creators when creating interactive applications that require precision in depth perception (e.g. gaming, surgery training).
L. Watkin, G. Barnaby, and A. Roudaut

\section{ACKNOWLEDGMENTS}

This work was supported by the Engineering and Physical Sciences Research Council (grant number EPSRC EP/P00s4342/1) and the Leverhulme Trust.

\section{REFERENCES}

1. Al Maimani, A., Roudaut, A. (2017, May). Frozen suit: designing a changeable stiffness suit and its application to haptic games. In Proceedings of the $2017 \mathrm{CHI}$ Conference on Human Factors in Computing Systems (pp. 2440-2448). ACM.

2. Armbrüster, C., Wolter, M., Kuhlen, T., Spijkers, W., \& Fimm, B. (2008). Depth perception in virtual reality: distance estimations in peri-and extrapersonal space. Cyberpsychology \& Behavior, 11(1), 9-15.

3. Azmandian, M., Hancock, M., Benko, H., Ofek, E., \& Wilson, A. D. (2016, May). Haptic retargeting: Dynamic repurposing of passive haptics for enhanced virtual reality experiences. In Proceedings of the 2016 chi conference on human factors in computing systems (pp. 1968-1979). ACM

4. Barnaby G., Roudaut A., Mantis: A Scalable, Lightweight and Accessible Architecture to Build Multiform Force Feedback Systems. In Proceedings of the $32^{\text {nd }}$ ACM Symposium on User Interface Software and Technology UIST'19.

5. Borro, D., Savall, J., Amundarain, A., Gil, J. J., Garcia-Alonso, A., \& Matey, L. (2004). A large haptic device for aircraft engine maintainabil[21]ity. IEEE Computer Graphics and Applications, 24(6), 70-74.

6. Bouguila, L., Ishii, M., \& Sato, M. (2000, September). Effect of coupling haptics and stereopsis on depth perception in virtual environment. In Proc. of the 1st Workshop on Haptic Human Computer Interaction (pp. 54-62).

7. Cooper, N., Milella, F., Pinto, C., Cant, I., White, M., \& Meyer, G. (2018). The effects of substitute multisensory feedback on task performance and the sense of presence in a virtual reality environment. PloS one, 13(2), e0191846.

8. Finnegan, D. J., O'Neill, E., \& Proulx, M. J. (2017, March). An approach to reducing distance compression in audiovisual virtual environments. In 2017 IEEE 3rd VR Workshop on Sonic Interactions for Virtual Environments (SIVE) (pp. 1-6). IEEE.

9. Gerig, N., Mayo, J., Baur, K., Wittmann, F., Riener, R., \& Wolf, P. (2018). Missing depth cues in virtual reality limit performance and quality of three dimensional reaching movements. PLoS one, 13(1), e0189275.

10. Grossman, T., \& Balakrishnan, R. (2006, May). An evaluation of depth perception on volumetric displays. In Proceedings of the working conference on Advanced visual interfaces (pp. 193-200). ACM.

11. Hecht, D., \& Reiner, M. (2009). Sensory dominance in combinations of audio, visual and haptic stimuli. Experimental brain research, 193(2), 307-314.

12. Jones, J. A., Swan II, J. E., Singh, G., Kolstad, E., \& Ellis, S. R. (2008, August). The effects of virtual reality, augmented reality, and motion parallax on egocentric depth perception. In Proceedings of the 5th symposium on Applied perception in graphics and visualization (pp. 9-14). ACM.

13. Kelly, J. W., Cherep, L. A., \& Siegel, Z. D. (2017). Perceived space in the HTC vive. ACM Transactions on Applied Perception (TAP), 15(1), 2.

14. Kruijff, E., Swan, J. E., \& Feiner, S. (2010, October). Perceptual issues in augmented reality revisited. In 2010 IEEE International Symposium on Mixed and Augmented Reality (pp. 3-12). IEEE.

15. Lawson, G., Roper, T., \& Abdullah, C. (2016, September). Multimodal Sensory Illusions for Improving Spatial Awareness in Virtual Environments. In Proceedings of the European Conference on Cognitive Ergonomics (p. 14). ACM. 
16. Lin, Q., Xie, X., Erdemir, A., Narasimham, G., McNamara, T. P., Rieser, J., \& Bodenheimer, B. (2011, August). Egocentric distance perception in real and HMD-based virtual environments: the effect of limited scanning method. In Proceedings of the ACM SIGGRAPH Symposium on Applied Perception in Graphics and Visualization (pp. 75-82). ACM.

17. Lunghi, C., Binda, P., \& Morrone, M. C. (2010). Touch disambiguates rivalrous perception at early stages of visual analysis. Current Biology, 20(4), R143-R144.

18. Naceri, A., Chellali, R., \& Hoinville, T. (2011). Depth perception within peripersonal space using head-mounted display. Presence: Teleoperators and Virtual Environments, 20(3), 254-272.

19. Omirou, T., Perez, A. M., Subramanian, S., \& Roudaut, A. (2016, March). Floating charts: Data plotting using free-floating acoustically levitated representations. In 2016 IEEE Symposium on 3D User Interfaces (3DUI) (pp. 187-190). IEEE.

20. Renner, R. S., Velichkovsky, B. M., \& Helmert, J. R. (2013). The perception of egocentric distances in virtual environments-a review. ACM Computing Surveys (CSUR), 46(2), 23.
21. Ries, B., Interrante, V., Anderson, L., \& Lindquist, J. (2006, July). Presence, rather than prior exposure, is the more strongly indicated factor in the accurate perception of egocentric distances in real world co-located immersive virtual environments. In ACM SIGGRAPH 2006 Research posters (p. 191). ACM.

22. Rolland, J. P., Gibson, W., \& Ariely, D. (1995). Towards quantifying depth and size perception in virtual environments. Presence: Teleoperators \& Virtual Environments, 4(1), 24-49.

23. Rosa, N., Hürst, W., Vos, W., \& Werkhoven, P. (2015, November). The Influence of visual cues on passive tactile sensations in a multimodal immersive virtual environment. In Proceedings of the 2015 ACM on International Conference on Multimodal Interaction (pp. 327-334). ACM.

24. Sato, M. (2002, September). Development of string-based force display: SPIDAR. In 8th international conference on virtual systems and multimedia.

25. Siegel, Z. D., \& Kelly, J. W. (2017). Walking through a virtual environment improves perceived size within and beyond the walked space. Attention, Perception, \& Psychophysics, 79(1), 39-44. 